PAREIGIS, B.

Math. Annalen 153, I-13 (1964)

\title{
Einige Bemerkungen über Frobenius-Erweiterungen
}

\author{
Von
}

Bono Pareigis* in Ithaca, N.Y.

1. Der von F. KASCH eingeführte Begriff der Frobenius-Erweiterung [2] konnte von T. NakaYaMa, T. TsuzukU und F. Kasch ([5], [8], [9]) weitgehend verallgemeinert werden, und eine in [2] angegebene Charakterisierung einer Frobenius-Erweiterung durch ihren Endomorphismenring bleibt dabei im wesentlichen erhalten ([4], [5], [8], [9], [10]). Ferner gab F. KASCH [6] eine Definition des Nakayama-Automorphismus für eine beliebige FrobeniusErweiterung $R / S$, der jetzt ein Automorphismus des Zentralisators von $\mathbb{S}$ in $R$ ist.

Im ersten Teil dieser Note soll der Zusammenhang zwischen dem NakayamaAutomorphismus von $R / S$ und dem des Endomorphismenrings von $R / S$ untersucht werden. Es wird gezeigt, daß der Nakayama-Automorphismus beim Ubergang zum Endomorphismenring im wesentlichen erhalten bleibt.

Im zweiten Teil untersuchen wir folgende Frage: Gegeben seien unitäre Ringerweiterungen $R \supseteqq S \supseteqq T$, und seien zwei der drei Erweiterungen $R / S$, $R / T, S / T$ Frobenius-Erweiterungen. Ist dann auch die dritte eine FrobeniusErweiterung? In diesem Teil lassen sich durch explizite Angabe der NakayamaAutomorphismen gewisse Aussagen über den Fall von symmetrischen Frobenius-Erweiterungen machen.

Durch die Verallgemeinerung des Begriffes einer Frobenius-Erweiterung erhebt sich unter anderem die Frage, ob die Maschke-Ikeda-Kasch-Charakterisierung für relativ projektive bzw. relativ injektive Moduln erhalten bleibt. In [9] wird diese Frage unter gewissen zusätzlichen Voraussetzungen positiv beantwortet. Wie im Zusatz von [6] bemerkt wurde, ist es nach einer Idee von K. GruenBerg möglich, den Begriff der Spur auch für beliebige FrobeniusErweiterungen zu bilden. Im dritten Teil dieser Note soll das ausgeführt werden. Damit kann dann gezeigt werden, daß die Maschke-Ikeda-Kasch-Charakterisierung von relativ projektiven bzw. relativ injektiven Moduln ohne weitere Voraussetzungen für beliebige Frobenius-Erweiterungen gültig bleibt.

2. Alle in dieser Arbeit verwendeten Ringerweiterungen seien unitäre Ringerweiterungen, und alle Moduln seien unitäre Moduln. Nach [9] wollen wir im folgenden unter einer $\alpha$-Frobenius-Erweiterung bzw. unter einer Frobenius-Erweiterung zweiter Art $R / S$ eine Ringerweiterung $R \supseteq S$ mit den

* Während eines Teils der Arbeit unterstützt durch Nato-Forschungs-Stipendium 4-s-nato $2 / 3$ gf.

Math. Ann. 153 
Eigenschaften

(r1) $\varphi:{ }_{S} R_{R} \cong{ }_{S}$ Hom $\left(R_{S}, S_{\alpha S}\right)_{R}$

(r2) $R_{S}$ ist endlich erzeugt und projektiv

bzw. den nach [9] dazu äquivalenten Eigenschaften

(11) $\varphi^{\prime}:{ }_{R} R_{S} \cong{ }_{R} \operatorname{Hom}\left({ }_{S} R, \alpha^{\prime} S_{S} S\right)_{S}$

(12) ${ }_{S} R$ ist endlich erzeugt und projektiv

verstehen. Dabei sei Hom $\left(R_{S}, S_{\alpha, S}\right)$ die Menge der Homomorphismen von $R$ in $S$ mit $f(r s)=f(r) \alpha(s)$ für $r \in R, s \in S$, wobei $\alpha$ ein Automorphismus von $S$ sei. $\varphi$ bzw. $\varphi^{\prime}$ werden Frobenius-Isomorphismen genannt. Ist $\alpha$ die identische Abbildung, so sagen wir, $R / S$ ist eine 1-Frobenius-Erweiterung oder eine Frobenius-Erweiterung erster Art. Gelten an Stelle von (r2) bzw. (12) die Eigenschaften bzw.

(r3) $R_{S}$ ist endlich erzeugt und frei

(13) ${ }_{S} R$ ist endlich erzeugt und frei, so heißt die Frobenius-Erweiterung frei.

Wir schreiben Homomorphismen von links und Multiplikatoren werden in den folgenden vier Möglichkeiten vorkommen:

a) $\left[r^{l} f\right](a)=f(r a)$

b) $\left[{ }^{l} r f\right](a)=r f(a)$

c) $\left[r^{r} f\right](a)=f(a r)$

d) $[r r f](a)=f(a) r$

wobei $r \in R, a \in{ }_{R} A$ bzw. $A_{R}, f \in \operatorname{Hom}(A, B)$ und $B$ ein $R$-Rechts- bzw. $R$ Links-Modul sind. In den Fällen b) und c) ist die Menge der so definierten Operatoren ring-isomorph zu $R$, in den Fällen a) und d) zu $R^{0}$, dem zu $R$ inversisomorphen Ring.

Nach [5] und [9] können (r1) bzw. (11) ersetzt werden durch die dazu äquivalenten Eigenschaften

(r1') Es gibt einen Homomorphismus $\psi \in \operatorname{Hom}\left({ }_{S} R_{S},{ }_{S} S_{\alpha S}\right)$ so, daß die Abbildung

$$
R \ni r \rightarrow\left[r^{l} \psi\right] \in \operatorname{Hom}\left(R_{S}, S_{\alpha, S}\right)
$$

ein $S$ - $R$-Isomorphismus ist.

bzw.

$\left(11^{\prime}\right)$ Es gibt einen Homomorphismus $\psi^{\prime} \in \operatorname{Hom}\left({ }_{S} R_{S}, \alpha^{\prime} S_{S} S_{S}\right)$ so, daß die Abbildung

ein $R$-S-Isomorphismus ist.

$$
R \ni r \rightarrow\left[r^{r} \psi^{\prime}\right] \in \operatorname{Hom}\left({ }_{S} R,{ }_{\alpha^{\prime}} S\right)
$$

Dabei gilt $\psi=\varphi(1), \psi^{\prime}=\varphi^{\prime}(1), \psi^{\prime}=\alpha^{-1} \psi, \alpha^{\prime}=\alpha^{-1}$ für jeweils zusammengehörige Abbildungen. Die Homomorphismen $\psi$ und $\psi^{\prime}$ heißen FrobeniusHomomorphismen. 
Seien $\varrho, \varrho^{\prime} \in Z_{R}(S)=P$, dem Zentralisator von $S$ in $R$. Wegen ( $1^{\prime}$ ) ist $\left[\varrho^{l} \psi\right] \in \operatorname{Hom}\left(R_{S}, S_{\alpha S}\right)$ und, wovon man sich leicht überzeugt, sogar aus $\operatorname{Hom}\left({ }_{S} R_{S},{ }_{S} S_{\alpha S}\right)$. Dann ist $\alpha^{-1}\left[\varrho^{l} \psi\right]=\left[\varrho^{l} \alpha^{-1} \psi\right]=\left[\varrho^{l} \psi^{\prime}\right] \in \operatorname{Hom}\left({ }_{S} R_{S}, \alpha^{\prime} S^{S} S_{S}\right)$. Andererseits können alle Elemente aus $\operatorname{Hom}\left({ }_{S} R_{S}, \alpha^{\prime} S_{S} S_{S}\right)$ in der Form [ $\left.\varrho^{\prime r} \psi^{\prime}\right]$ dargestellt werden. Die Zuordnung $\psi^{*}: \varrho \rightarrow \varrho^{\prime}$ ist ein Ringautomorphismus von $P$, wie man leicht sieht. Dieser Automorphismus wird nach [6] NakayamaAutomorphismus genannt.

Wir wollen im folgenden die durch die oben vorgenommenen Konstruktionen zusammenhängenden Homomorphismen $\varphi, \psi$ und $\psi^{*}$ (eventuell auch $\varphi^{\prime}$ und $\psi^{\prime}$ ) als zusammengehörig bezeichnen. Es gilt nach Definition des NakayamaAutomorphismus immer

$$
\begin{aligned}
\psi(\varrho r) & =\psi\left(r \psi^{*}(\varrho)\right) \\
{\left[\varrho^{l} \psi\right] } & =\left[\psi^{*}(\varrho)^{r} \psi\right] \\
\varphi(\varrho r) & =\left[\psi^{*}(\varrho)^{r} \varphi(r)\right] \\
\varphi^{\prime}(r \varrho) & =\left[\psi^{*-1}(\varrho)^{l} \varphi^{\prime}(r)\right] .
\end{aligned}
$$

Um die Menge aller Nakayama-Automorphismen zu untersuchen, beachten wir, daß wegen (r l') $\psi^{*}$ von $\alpha$ abhängt. Es liegt die Frage nahe, wie der Auto- . morphismus $\alpha$ zu einem Automorphismus $\alpha^{\prime}$ abgeändert werden kann, damit wieder ein (r1) genügender Isomorphismus existiert. T. NAKAYAMA und T. Tsuzuku zeigen dazu [9]:

Sei $R / S$ eine $\alpha$-Frobenius-Erweiterung, und sei $\alpha^{\prime}$ ein weiterer Auto-

morphismus von $S$. Dann und nur dann ist $R / S$ eine $\alpha^{\prime}$-Frobenius-

Erweiterung, wenn der Automorphismus $\alpha^{\prime} \alpha^{-1}$ von $S$ von einem inneren

Automorphismus von $R$ induziert wird.

Man überzeugt sich leicht, daß der neu entstehende Frobenius-Homomorphismus $\left[\left(t^{-1}\right)^{l} \alpha^{\prime} \alpha^{-1} \psi\right]$ beim Übergang von $\alpha \mathrm{zu} \alpha^{\prime}$ ist, wobei $\alpha^{\prime} \alpha^{-1}(s)=t s t^{-1}$ $=\beta(s)$. Der dazugehörige Nakayama-Automorphismus ist dann $\psi^{*} \beta^{-1}$, denn es ist für $\varrho \in P$ und $r \in R$

$$
\begin{aligned}
{\left[\left(t^{-1}\right)^{l} \alpha^{\prime} \alpha^{-1} \psi\right](\varrho r) } & =\left(\alpha^{\prime} \alpha^{-1} \psi\right)\left(t^{-1} \varrho r\right) \\
& =\left(\alpha^{\prime} \alpha^{-1} \psi\right)\left(\beta^{-1}(\varrho) t^{-1} r\right) \\
& =\left(\alpha^{\prime} \alpha^{-1} \psi\right)\left(t^{-1} r\left(\psi^{*} \beta^{-1}\right)(\varrho)\right) \\
& =\left[\left(t^{-1}\right)^{l} \alpha^{\prime} \alpha^{-1} \psi\right]\left(r\left(\psi^{*} \beta^{-1}\right)(\varrho)\right) .
\end{aligned}
$$

Einen Uberblick über die Gesamtheit der möglichen zusammengehörigen Abbildungen $\varphi, \psi$ und $\psi^{*}$ bei festem $\alpha$ gibt der

Satz 1. Man erhält aus zusammengehörigen Abbildungen $\varphi, \psi$ und $\psi^{*}$ genau alle weiteren zusammengehörigen Abbildungen $\varphi^{+}, \psi^{+}$und $\left(\psi^{*}\right)^{+}$in der Form $\left[\varrho^{l} \varphi\right],\left[\varrho^{l} \psi\right]$ und $\left[\varrho^{l}\left(\varrho^{-1}\right)^{r} \psi^{*}\right]$, wobei $\varrho \in P^{*}$ ist, der multiplikativen Gruppe der regulären Elemente von $P$.

Der Beweis für $\varphi$ weicht von den bekannten Beweisen nicht ab, und der Beweis für $\psi$ und $\psi^{*}$ folgt aus den oben angegebenen Konstruktionen. Es sei darauf hingewiesen, daß $\left[\varrho^{l}\left(\varrho^{-1}\right)^{r} \psi^{*}\right]$ bedeutet, daß sich $\psi^{*}$ um einen inneren Automorphismus von $P$ ändert. 
Wir wollen eine Frobenius-Erweiterung zweiter Art symmetrisch nennen, wenn ein Nakayama-Automorphismus die identische Abbildung ist. Wegen Satz 1 und (2) sind dazu folgende Aussagen äquivalent:

1) Ein Nakayama-Automorphismus wird induziert von einem inneren Automorphismus $\beta$ von $R$, für den gilt $\beta(S)=S$.

2) Jeder Nakayama-Automorphismus wird induziert von einem inneren Automorphismus $\beta$ von $R$, für den gilt $\beta(S)=S$.

Weiter wollen wir eine $\alpha$-Frobenius-Erweiterung $\alpha$-symmetrisch nennen, wenn ein mit einem $S-\propto S$-Frobenius-Homomorphismus zusammenhängender Nakayama-Automorphismus die identische Abbildung ist. Wegen Satz 1 sind dazu folgende Aussagen äquivalent:

1) Ein mit einem $S-\alpha S$-Frobenius-Homomorphismus zusammengehöriger Nakayama-Automorphismus ist ein innerer Automorphismus von $P$.

2) Jeder mit einem $\boldsymbol{S}-\alpha \boldsymbol{S}$-Frobenius-Homomorphismus zusammengehörige Nakayàma-Automorphismus ist ein innerer Automorphismus von $P$.

Da der mit vorgegebenen $\varphi, \varphi^{\prime}$ bzw. $\psi$ zusammengehörige NakayamaAutomorphismus durch jede der vier Gleichungen (1) eindeutig bestimmt ist, haben wir damit eine Vielzahl untereinander äquivalenter Kennzeichnungen für symmetrische bzw, $\alpha$-symmetrische $\alpha$-Frobenius-Erweiterungen.

3. Wir wollen in diesem Teil den Zusammenhang zwischen dem NakayamaAutomorphismus der $\alpha$-Frobenius-Erweiterung $R / S$ und dem des Endomorphismenringes von $R / S$ untersuchen. Dazu geben wir die Sätze über den Endomorphismenring aus [5] für $\alpha$-Frobenius-Erweiterungen an.

Satz 2. Sei R/S eine $\beta$-Frobenius-Erweiterung und lasse sich $\beta$ fortsetzen zu einem Automorphismus $\alpha$ von $R$. Dann ist $\operatorname{Hom}\left(R_{S}, R_{S}\right) /\left[{ }^{l} R\right.$ id] eine $\alpha^{-1}$. Frobenius-Erweiterung.

Eine Verallgemeinerung des Beweises aus [5] ist ohne weiteres möglich, wenn man beachtet, daß man als zweiseitigen $R$-Homomorphismus in [5]

erhält, für den gilt, daß

$$
\Psi_{1}:{ }_{R} \operatorname{Hom}\left(R_{S}, R_{\beta S}\right)_{R} \rightarrow{ }_{R} R_{R}
$$

ein Isomorphismus ist.

$$
\operatorname{Hom}\left(R_{S}, R_{\beta S}\right) \ni f \rightarrow\left[f^{r} \Psi_{1}\right] \in \operatorname{Hom}\left({ }_{R} \operatorname{Hom}\left(R_{S}, R_{\beta S}\right),{ }_{R} R\right)
$$

$\Psi_{1}$ ist in diesem Fall noch nicht der gesuchte Frobenius-Homomorphismus des Endomorphismenrings. Diesen erhalten wir, wenn wir bilden

$$
\Psi_{2}=\left[\alpha^{l} \Psi_{1}\right]
$$

Dann ist

$$
\Psi_{2}:{ }_{R} \operatorname{Hom}\left(R_{S}, R_{S}\right)_{R} \rightarrow{ }_{\alpha R} R_{R}
$$

ein $\left(11^{\prime}\right)$ genügender Homomorphismus, was zu zeigen war. Es sei weiter bemerkt, daß bei dem Beweis in [5] folgender Zusammenhang zwischen $\Psi_{1}$ und dem Frobenius-Isomorphismus $\varphi$ von $R / S$ besteht. Sei $f \in \operatorname{Hom}\left(R_{S}, S_{\beta S}\right)$, so ist

$$
\Psi_{1}(f)=\varphi^{-1}(f) \text {. }
$$

Die Umkehrung des Satzes wird von F. KASCH [5] unter der Voraussetzung bewiesen, $\operatorname{daB} R_{S}=x S_{S} \oplus A_{S}$ und $x S_{S} \cong S_{S}$ für 1-Frobenius-Erweiterungen. 
T. Nakayama und T. Tsuztro zeigten in [10], daB ohne diese Voraussetzung der Satz nicht gilt. Auch dieser Satz läßt sich ohne weiteres für $\alpha$-FrobeniusErweiterungen beweisen.

Satz 3. Sei $R / S$ eine Ringerweiterung, genüge $\operatorname{Hom}\left(R_{S}, R_{S}\right) /\left[{ }^{l} R\right.$ id $]$ der Bedingung $\left(11^{\prime}\right)$ und sei $\alpha(S)=S$. Dann gibt es einen $S$ - $R$-Monomorphismus von $\operatorname{Hom}\left(R_{S}, S_{\alpha S}\right)$ in $R$. Ist außerdem $R_{S}=x S_{S} \oplus A_{S}$ und $x S_{S} \cong S_{S}$, so existiert ein $S$-R-Isomorphismus zwischen $\operatorname{Hom}\left(R_{S}, S_{\alpha S}\right)$ und $R$, d.h. dann ist (r 1) für $R / S$ erfüllt.

Zum Beweis verweisen wir auf [5].

Wir wollen im folgenden, da [ $\left.{ }^{l} R \mathrm{id}\right]$ und $R$ als Ringe isomorph sind, [ $\left.{ }^{l} R \mathrm{id}\right]$ durch $R$ ersetzen. Ehe wir den Nakayama-Automorphismus angeben, der zu der Frobenius-Erweiterung $\operatorname{Hom}\left(R_{S}, R_{S}\right) / R$ gehört, muß der Zentralisator von $R$ in $\operatorname{Hom}\left(R_{S}, R_{S}\right)$ untersucht werden. Es ist

$$
Z_{\text {Hom }\left(R_{S}, R_{S}\right)}(R)=\operatorname{Hom}\left({ }_{R} R_{S},{ }_{R} R_{S}\right) .
$$

Weiter ist mit $f \in \operatorname{Hom}\left({ }_{R} R_{S},{ }_{R} R_{S}\right) f(a)=\left[{ }^{r} f(1)\right.$ id] $(a)$ und wegen $s f(1)=f(1) s$ für $s \in S$ ist $f(1) \in P=Z_{R}(S)$. Weiter ist für jedes $\varrho \in P\left[{ }^{r} \varrho\right.$ id] $\in \operatorname{Hom}\left({ }_{R} R_{S},{ }_{R} R_{S}\right)$. Also ist

$$
Z_{\text {Hom }\left(R_{S}, R_{s}\right)}(R)=\left[{ }^{r} P \mathrm{id}\right] .
$$

Satz 4. Sei R/S eine $\beta$-Frobenius-Erweiterung und lasse sich $\beta$ fortsetzen $z u$ einem Automorphismus $\alpha$ von $R$. Dann existiert eine eineindeutige Zuordnung zwischen den Frobenius-Homomorphismen $\Psi \in \operatorname{Hom}\left({ }_{R} \operatorname{Hom}\left(R_{S}, R_{S}\right)_{R}, \alpha R R_{R}\right)$ und den Frobenius-Isomorphismen $\varphi \in \operatorname{Hom}\left({ }_{S} \operatorname{Hom}\left(R_{S}, S_{\beta S}\right)_{R},{ }_{S} R_{R}\right)$. Weiter ist $\Psi^{*}\left(\left[{ }^{r} \varrho \mathrm{id}\right]\right)=\left[{ }^{\gamma}\left(\psi^{*} \alpha^{-1}(\varrho)\right)\right.$ id $]$ für $\varrho \in P$, und eine Änderung von $\psi^{*}$ um einen von $\varrho \in P^{*}$ erzeugten inneren Automorphismus von $P$ entspricht einer $\ddot{A}$ nderung von $\Psi^{*}$ um einen von $\left[r\left(\alpha\left(\varrho^{-1}\right)\right) \mathrm{id}\right]$ erzeugten inneren Automorphismus von [r $P$ id] .

Beweis: Wegen (4) und da sich nach Satz $1 \varphi$ und $\Psi$ genau um Elemente aus $P^{*}$ bzw. $\left[{ }^{r} P \mathrm{id}\right]^{*}=\left[{ }^{r} P^{*}\right.$ id] ändern können, ist die erste Behauptung klar. Falls $\Psi^{*}\left(\left[{ }^{r} \varrho\right.\right.$ id $\left.]\right)=\left[{ }^{r}\left(\psi^{*} \alpha^{-1}(\varrho)\right)\right.$ id $]$ ist, ist die dritte Behauptung klar, da $\left[{ }^{r} P\right.$ id $]$ invers-isomorph zu $P$ ist. Wegen $\varphi(\varrho r)=\left[\psi^{*}(\varrho)^{r} \varphi(r)\right]$ ist $\varphi^{-1}\left(\left[\psi^{*}\right.\right.$ $\left.\left.(\varrho)^{r} f\right]\right)=\varrho \varphi^{-1}(f)$. Sei $g \in \operatorname{Hom}\left(R_{S}, R_{\beta S}\right)$, so kann man schreiben $g=\sum\left[{ }^{l} r_{i} f_{i}\right]$ mit $f_{i} \in \operatorname{Hom}\left(R_{S}, S_{\beta S}\right)$. Dann ist $\Psi_{1}(g)=\sum r_{i} \varphi^{-1}\left(f_{i}\right)$ (siehe dazu [5]). Damit ist

$$
\begin{aligned}
& \Psi_{1}\left(\left[{ }^{r} \varrho \text { id }\right] g\right)=\Psi_{1}\left(\left[{ }^{r} \varrho\left(\sum{ }^{i} r_{i} f_{i}\right)\right]\right) \\
& =\Psi_{1}\left(\left[\sum{ }^{l} r_{i}{ }^{r} \varrho f_{i}\right]\right) \\
& =\Psi_{1}\left(\left[\sum{ }^{l} r_{i}{ }^{l} \varrho f_{i}\right]\right) \\
& =\sum r_{i} \varrho \varphi^{-1}\left(f_{i}\right) \\
& =\sum \cdot r_{i} \varphi^{-1}\left(\left[\psi^{*}(\varrho)^{r} f_{i}\right]\right) \\
& =\Psi_{1}\left(\left[\sum^{l} r_{i} \psi^{*}(\varrho)^{r} f_{i}\right]\right) \\
& =\Psi_{1}\left(\left[\psi^{*}(\varrho)^{r} \sum^{l} r_{i} f_{i}\right]\right) \\
& =\Psi_{1}\left(\left[\psi^{*}(\varrho)^{r} g\right]\right) \\
& =\Psi_{1}\left(g\left[{ }^{r}\left(\psi^{*}(\varrho)\right) \mathrm{id}\right]\right) \text {. }
\end{aligned}
$$


Wegen (3) ist dann

oder für $f \in \operatorname{Hom}\left(R_{S}, R_{S}\right)$

$$
\Psi_{2}\left(\left[{ }^{r}(\alpha(\varrho)) \mathrm{id}\right](\alpha g)\right)=\Psi_{2}\left((\alpha g)\left[{ }^{r}\left(\psi^{*}(\varrho)\right) \mathrm{id}\right]\right)
$$

$$
\Psi_{2}\left(\left[{ }^{r} \varrho \text { id }\right] f\right)=\Psi_{2}\left(f\left[{ }^{r}\left(\psi^{*} \alpha^{-1}(\varrho)\right) \mathrm{id}\right]\right),
$$

womit $\Psi^{*}\left(\left[{ }^{r} \varrho\right.\right.$ id $\left.]\right)=\left[r\left(\psi^{*} \alpha^{-1}(\varrho)\right)\right.$ id $]$ bewiesen ist.

Durch die Definition der $\alpha$-symmetrischen $\alpha$-Frobenius-Erweiterungen folgt aus Satz 4 sofort

Satz 5: Sei R/S eine 1-Frobenius-Erweiterung. R/S ist genau dann 1-symmetrisch, wenn $\operatorname{Hom}\left(R_{S}, R_{S}\right) / R$ 1-symmetrisch ist.

4. Wir untersuchen jetzt den Fall dreier Ringe $R \supseteq S \geqq T$. Sind $R / S$, $R / T$ bzw. $S / T$ Frobenius-Erweiterungen zweiter Art, so kennzeichnen wir die Abbildungen $\varphi, \psi$ und $\psi^{*}$ mit den Indices 1, 2 bzw. 3, und die Automorphismen aus (r1) sollen mit $\alpha, \beta$ bzw. $\gamma$ bezeichnet werden.

Satz 6: Seien $R \geqq S \geqq T$ Ringerweiterungen. Sei $S / T$ eine $\gamma$-FrobeniusErweiterung. Sei $R / S$ eine $\alpha$-Frobenius-Erweiterung, und sei $\alpha(T)=T$. Dann ist $R / T$ eine $\beta$-Frobenius-Erweiterung.

Beweis: Da $R_{S}$ und $S_{T}$ endlich erzeugt und projektiv sind, ist (r2) für $R_{T}$ erfüllt. Beachten wir, daß nach [1] II 6.4 gilt

$$
{ }_{T} \operatorname{Hom}\left(R_{S}, \operatorname{Hom}\left(S_{T}, T_{T}\right)_{S}\right)_{R} \cong{ }_{T} \operatorname{Hom}\left(R_{T}, T_{T}\right)_{R}
$$

und für jeden Automorphismus $\alpha$ von $S$ durch die Abbildung $\delta: \operatorname{Hom}\left(R_{S}, S_{S}\right) \ni$ $\exists f \rightarrow \alpha f \in \operatorname{Hom}\left(R_{S}, S_{\alpha S}\right)$ ein Isomorphismus

$$
{ }_{S} \operatorname{Hom}\left(R_{S}, S_{S}\right)_{R} \cong{ }_{\alpha S} \operatorname{Hom}\left(R_{S}, S_{\alpha, S}\right)_{R}
$$

erzeugt wird, wobei $\delta\left(\left[{ }^{l} r f\right]\right)=\left[{ }^{l}(\alpha(r)) \delta(f)\right]$ ist, so ist wegen $\alpha(T)=T$

$$
\begin{aligned}
& { }_{T} R_{R} \cong{ }_{T} \operatorname{Hom}\left(R_{S}, S_{\alpha S}\right)_{R} \\
& \cong{ }_{\alpha^{-1}} \operatorname{Hom}\left(R_{S}, S_{S}\right)_{R} \\
& \cong_{\alpha^{-1} T} \operatorname{Hom}\left(R_{S}, \operatorname{Hom}\left(S_{T}, T_{\gamma T}\right)_{S}\right)_{R} \\
& \cong \gamma^{-1} \alpha^{-1} T \operatorname{Hom}\left(R_{S}, \operatorname{Hom}\left(S_{T}, T_{T}\right)_{S}\right)_{R} \\
& \cong \gamma^{-1} \alpha^{-1} T \text { Hom }\left(R_{T}, T_{T}\right)_{R} \\
& \cong{ }_{\beta \gamma^{-1} \alpha^{-1}} T^{H} \operatorname{Hom}\left(R_{T}, T_{\beta T}\right)_{R} \text {. }
\end{aligned}
$$

Wählen wir $\beta=\alpha \gamma$, so ist damit auch (rl) nachgewiesen.

Bemerkung: Wegen Satz 2 und Satz 6 ist also $\operatorname{Hom}\left(R_{S}, R_{S}\right) / S$ eine 1-Frobenius-Erweiterung, wenn nur die Bedingungen von Satz 2 erfüllt sind.

Sei nun $R / S$ eine $\gamma$-Frobenius-Erweiterung und sei $S \leqq Z_{R}(R)$. Dann ist $\psi(\gamma(s) r)=\gamma(s) \psi(r)=\psi(r) \gamma(s)=\psi(r s)=\psi(s r)$, also ist $\gamma=$ id und $R / S$ eine 1-Frobenius-Erweiterung.

Satz 7: Seien $R \geqq S \geqq T$ Ringerweiterungen, und sei $S / T$ eine 1-FrobeniusErweiterung mit $T \leqq Z_{S}(S)$. Sei $R / T$ eine $\beta$-Frobenius- Erweiterung, und existiere ein Nakayama-Automorphismus $\psi_{2}^{*}$ so, daß $\psi_{2}^{*}(S)=S$. Sei $R_{S}$ projektiv. Dann ist $R / S$ eine $\alpha$-Frobenius-Erweiterung. 
Beweis: Da $R_{T}$ endlich erzeugt ist, ist auch $R_{S}$ endlich erzeugt, und damit ist (r 2) für $R_{S}$ erfüllt. Da $S / T$ und $R / T$ Frobenius-Erweiterungen sind und wegen [1] II 6.4 und wegen (6), ist

$$
\begin{aligned}
R_{R} & \cong \operatorname{Hom}\left(R_{T}, T_{\beta T}\right)_{R} \\
& \cong \operatorname{Hom}\left(R_{T}, T_{T}\right)_{R} \\
& \cong \operatorname{Hom}\left(R_{S}, \operatorname{Hom}\left(S_{T}, T_{T}\right)_{S}\right)_{R} \\
& \cong \operatorname{Hom}\left(R_{S}, S_{S}\right)_{R} \\
& \cong \operatorname{Hom}\left(R_{S}, S_{\alpha S}\right)_{R} .
\end{aligned}
$$

Setzen wir $\alpha=\psi_{2}^{*-1} \psi_{3}^{*}$, was wegen $\psi_{2}^{*}(S)=S$ möglich ist, so ist mit $r \in R$

$$
\varphi_{1}(r)=\left[r^{l} \propto \varphi_{3}^{-1} \tilde{\beta}^{-1} \varphi_{2}^{\prime}\right] \text {. }
$$

Dabei sei $\tilde{\beta}$ der von $\beta$ induzierte Isomorphismus

$$
\operatorname{Hom}(1, \beta): \operatorname{Hom}\left(R_{T}, T_{T}\right) \rightarrow \operatorname{Hom}\left(R_{T}, T_{\beta T}\right)
$$

und $\varphi_{2}^{\prime}(r)$ die Einschränkung von $\varphi_{2}(r)$ auf $S$.

Bevor wir beweisen, daß $\varphi_{1}$ ein $S$ - $R$-Isomorphismus ist, müssen wir einige Vorbemerkungen machen. Wegen $\psi_{2}^{*}(S)=S$ ist auch $\psi_{2}^{*}\left(Z_{R}(S)\right)=Z_{R}(S)$. Wir betrachten $\varrho \in Z_{R}(T), r \in R$ und $s \in S$. Wegen (1) ist

$$
\left[\varrho^{l} \varphi_{2}^{\prime}\right](r)=\left[\psi_{2}^{*}(\varrho)^{r} \varphi_{2}(r)\right] \text {. }
$$

Damit (7) auch für Elemente $s \in S$ anstelle von $\varrho \in Z_{R}(T)$ gilt, muß $S \leqq Z_{R}(T)$ sein. Hier geht also $T \subseteq Z_{S}(S)$ ein.

Seien $r \in R, s, s^{\prime} \in S, f \in \operatorname{Hom}\left(S_{T}, T_{T}\right)$, dann ist wegen

d. h. wegen

$$
\varphi_{3}\left(s s^{\prime}\right)=\left[\psi_{2}^{*}(s)^{r} \varphi_{\mathbf{3}}\left(s^{\prime}\right)\right],
$$

auch

$$
\left[{ }^{l} s \varphi_{3}^{-1}(f)\right]=\varphi_{3}^{-1}\left(\left[\psi_{3}^{*}(s)^{r} f\right]\right)
$$

$$
\left.{ }^{l} s \varphi_{3}^{-1} \tilde{\beta}^{-1} \varphi_{2}^{\prime}\right](r)=\varphi_{3}^{-1}\left(\left[\psi_{3}^{*}(s)^{r} \tilde{\beta}^{-1} \varphi_{2}^{\prime}(r)\right]\right) .
$$

Mit diesen Hilfsmitteln können wir für $r \in R, s \in S$ zeigen

$$
\begin{aligned}
\varphi_{1}(s r) & =\left[r^{l} s^{l} \alpha \varphi_{3}^{-1} \tilde{\beta}^{-1} \varphi_{2}^{\prime}\right] \\
& =\left[r^{l} \alpha\left(\varphi_{3}^{-1} \tilde{\beta}^{-1}\left[s^{l} \varphi_{2}^{\prime}\right]\right)\right] \\
& =\left[r^{l} \alpha\left[{ }^{l}\left(\psi_{3}^{*-1} \psi_{2}^{*}(s)\right) \varphi_{3}^{-1} \tilde{\beta}^{-1} \varphi_{2}^{\prime}\right]\right] \quad \text { wegen (7) und (8) } \\
& =\left[{ }^{l} s \varphi_{1}(r)\right],
\end{aligned}
$$

also ist $\varphi_{1}$ ein $S$-Links-Isomorphismus.

Sei jetzt $\varrho \in Z_{R}(S)$, so ist wegen

$$
\begin{aligned}
{\left[\varrho^{l} \varphi_{2}^{\prime}\right](r)(s) } & =\psi_{2}(\varrho r s) \\
& =\psi_{2}\left(r s \psi_{2}^{*}(\varrho)\right) \\
& =\psi_{2}\left(r \psi_{2}^{*}(\varrho) s\right) \\
& =\left[\psi_{2}^{*}(\varrho)^{r} \varphi_{2}^{\prime}\right](r)(s)
\end{aligned}
$$


also

$$
\left[\varrho^{l} \psi_{2}^{\prime}\right]=\left[\psi_{2}^{*}(\varrho)^{r} \varphi_{2}^{\prime}\right]
$$

Wir wollen den Zusammenhang zwischen den Nakayama-Automorphismen $\psi_{1}^{*}$ und $\psi_{2}^{*}$ untersuchen. Da $\psi_{1}^{*}$ auf $Z_{R}(S)$ und $\psi_{2}^{*}$ auf $Z_{R}(T)$ operieren, ist $\psi_{1}^{*}$ Einschränkung von $\psi_{2}^{*}$ auf $Z_{R}(S)$. Es ist nämlich mit $\varrho \in Z_{R}(S)$ und $r \in R$

$$
\begin{aligned}
\varphi_{1}(\varrho r) & =\left[r^{l} \varrho^{l} \alpha \varphi_{3}^{-1} \tilde{\beta}^{-1} \varphi_{2}^{\prime}\right] \\
& =\left[r^{l} \alpha \varphi_{3}^{-1} \tilde{\beta}^{-1}\left[\varrho^{l} \varphi_{2}^{\prime}\right]\right] \\
& =\left[r^{l} \alpha \varphi_{3}^{-1} \tilde{\beta}^{-1}\left[\psi_{2}^{*}(\varrho)^{r} \varphi_{2}^{\prime}\right]\right] \text { wegen (9) } \\
& =\left[\psi_{2}^{*}(\varrho)^{r} \varphi_{1}(r)\right] .
\end{aligned}
$$

Durch Spezialisierung auf symmetrische Frobenius-Erweiterungen erhalten wir den

Satz 8: Seien $R \geqq S \geqq T$ Ringerweiterungen. Sei $S / T$ eine 1-FrobeniusErweiterung, und sei $T \leqq Z_{S}(S)$. Sei $R_{S}$ projeltiv. Ist $R / T$ eine symmetrische $\beta$-Frobenius-Erweiterung, so ist $R / S$ eine symmetrische $\alpha$-Frobenius-Erweiterung

Zusatz: Sind $S / T$ und $R / T$ 1-symmetrische 1-Frobenius-Erweiterungen und gelten die Voraussetzungen von Satz 8 , so ist R/S eine 1-symmetrische 1 Frobenius-Erweiterung.

Mit diesem Zusatz können wir ein Beispiel für eine 1-symmetrische 1Frobenius-Erweiterung $R / S$ angeben, bei der $S$ nicht kommutativ ist. Seien nämlich $R$ und $S$ halbeinfache Algebren über einem Körper $T$. Dann ist $R / S$ eine 1-symmetrische 1-Frobenius-Erweiterung, denn jede halbeinfache Algebra über einem Körper ist eine 1-symmetrische 1-Frobenius-Erweiterung. Weiter ist $R_{S}$ projektiv, da $S$ halbeinfach ist. Damit sind die Voraussetzungen des Zusatzes erfüllt.

Ein weiteres Beispiel für eine 1-symmetrische 1-Frobenius-Erweiterung ist folgendes : Sei $R$ ein kommutativer Ring, $G$ eine Gruppe endlicher Ordnung und $H$ eine Untergruppe von $G$. Dann ist für die Gruppenringe $R[G] / R[H]$ eine 1-symmetrische 1-Frobenius-Erweiterung. Es ist nämlich $R[G] / R$ eine 1-symmetrische 1-Frobenius-Erweiterung. Daher gilt auch hier der Zusatz von Satz 8.

Satz 9: Seien $R \supseteq S \geqq T$ Ringerweiterungen. Seien $R / T$ eine $\beta$-FrobeniusErweiterung, $S_{T}$ endlich erzeugt und projektiv,

und

$$
T^{R_{S}}=\bigoplus_{i=1}^{n} x^{x_{i}} S_{S} \quad \text { mit } \quad T^{x_{i}} S_{S} \cong T S_{S}
$$

$$
{ }_{S} R_{T}=\stackrel{\oplus}{\oplus=1}_{S} S y_{i T} \quad \text { mit } \quad{ }_{S} S y_{i T} \cong S_{S} S_{T}
$$

und erfülle ${ }_{2} S_{S}$ Minimal- und Maximal-Bedingung. Dann ist $S / T$ eine $\beta$ Frobenius-Erweiterung. 
Beweis: Es ist

$$
\begin{aligned}
\stackrel{\oplus}{\oplus}_{i=1}^{n} T^{x_{i}} S_{S} & ={ }_{T} R_{S} \\
& \cong{ }_{T} \operatorname{Hom}\left(R_{T}, T_{\beta T}\right)_{S} \\
& \cong{ }_{T} \operatorname{Hom}\left(\stackrel{n}{\oplus}_{i=1} S y_{i T}, T_{\beta T}\right)_{S} \\
& \cong{ }_{i=1}^{n}{ }_{T} \operatorname{Hom}\left(S y_{i T}, T_{\beta T}\right)_{S}
\end{aligned}
$$

Da ${ }_{T} S_{S}$ Minimal- und Maximal-Bedingung erfüllt, erfüllt auch ${ }_{T} R_{S}$ Minimalund Maximal-Bedingung, also ist nach dem Satz von REMAK-KRULL-SchmidT ${ }_{T} S_{S} \cong{ }_{T}$ Hom $\left(S_{T}, T_{\beta T}\right)_{S}$ und, da nach Voraussetzung $S_{T}$ endlich erzeugt und projektiv ist, ist $S / T \beta$-Frobenius-Erweiterung.

Aus Satz 9 folgt unmittelbar der

Zusatz: Seien $R \supseteq S \geqq T$ Ringerweiterungen, $R / T$ eine $\beta$-FrobeniusErweiterung, $R / S$ eine freie $\alpha$-Frobenius-Erweiterung, $S$ ein Artinscher Ring, $T \subseteq Z_{R}(R)$ und $S_{T}$ endlich erzeugt und projektiv. Dann ist $S / T$ eine $\beta$-FrobeniusErweiterung.

5. K. GRUEnberg wies auf eine mögliche Verallgemeinerung der Spur hin (s. Zusatz in $\left.[6]^{1}\right)$ ). Betrachten wir eine $\beta$-Frobenius-Erweiterung $R / S$, so ist bekanntlich für jeden $S$-Links-Modul $A$

und damit

$$
\operatorname{Hom}\left({ }_{S} \operatorname{Hom}\left(R_{S}, S_{S}\right),{ }_{S} A\right) \cong R \underset{S}{\otimes} A
$$

$$
\operatorname{Hom}\left({ }_{S} \operatorname{Hom}\left(R_{S}, S_{\beta S}\right),{ }_{\beta^{-1} S} A\right) \cong R \underset{S}{\otimes} A
$$

Setzen $\operatorname{wir}_{\beta^{-1} S^{S}} A={ }_{S} R$, d. h. ${ }_{S} A={ }_{\beta S} R$, so erhalten wir

$$
\alpha: \operatorname{Hom}\left({ }_{S} \operatorname{Hom}\left(R_{S}, S_{\beta S}\right),{ }_{S} R\right) \cong R_{S} \otimes_{\beta S} R \text {, }
$$

wobei $\alpha^{-1}\left(r^{\prime} \otimes r^{\prime \prime}\right)(f)=f\left(r^{\prime}\right) r^{\prime \prime}$ ist. $R_{S} \otimes{ }_{\beta S} R$ bedeute dabei, daß statt $r^{\prime} s \otimes r^{\prime \prime}$ $=r^{\prime} \otimes s r^{\prime \prime}$ gelten soll $r^{\prime} s \otimes r^{\prime \prime}=r^{\prime} \otimes \beta(s) r^{\prime \prime}$. Wir zeigen, daß für freie $\beta$ Frobenius-Erweiterungen gilt

$$
\alpha\left(\varphi^{-1}\right)=\sum r_{i} \otimes l_{i} \in R_{S} \otimes \beta S,
$$

wobei $\left\{r_{i}\right\}$ bzw. $\left\{l_{i}\right\}$ die zueinander dualen Rechts- bzw. Links-Basen von $R / S$ sind. Es ist nämlich

$$
\begin{aligned}
\alpha^{-1}\left(\sum r_{i} \otimes l_{i}\right)(f) & =\sum f\left(r_{i}\right) l_{i} \\
& =\sum\left[r^{l} \psi\right]\left(r_{i}\right) l_{i} \\
& =\sum \psi\left(r r_{i}\right) l_{i} \\
& =\sum \psi\left(r_{i}\right) l_{i} r \\
& =r \\
& =\varphi^{-1}(f) .
\end{aligned}
$$

1) Ein etwas allgemeineres Ergebnis als das von Satz 11 wurde von K. GRUENBERG in einer bisher unveröffentlichten Arbeit bewiesen. 
Auch für die quasifreien $\beta$-Frobenius-Erweiterungen aus [9] gilt diese Beziehung wegen [9] Prop. 11 und [9] (40). Sei für beliebige $\beta$-Frobenius-Erweiterungen im folgenden $\alpha\left(\varphi^{-1}\right)=\sum r_{i} \otimes l_{i}$. Wir wollen sehen, welche Eigenschaften der $\left\{r_{i}\right\}$ und $\left\{l_{i}\right\}$ eine Frobenius-Erweiterung eindeutig bestimmen.

Satz 10: Sei $R \supseteqq S$ eine Ringerweiterung. $R / S$ ist dann und nur dann eine $\beta$-Frobenius-Erweiterung, wenn in $R$ Elementensysteme $\left\{r_{i}\right\}$ und $\left\{l_{i}\right\} i=1, \ldots, n$ und $\psi \in \operatorname{Hom}\left(R_{S}, S_{\beta S}\right)$ und $\psi^{\prime} \in \operatorname{Hom}\left({ }_{S} R_{S}, \beta^{-1} S_{S} S_{S}\right)$ derart existieren, daß

1) $\sum r r_{i} \otimes l_{i}=\sum r_{i} \otimes l_{i} r$ für $\sum r_{i} \otimes l_{i} \in R_{S} \otimes_{\beta S} R$ und alle $r \in R$

2) $\sum \psi\left(r_{i}\right) l_{i}=1=\sum r_{i} \psi^{\prime}\left(l_{i}\right)$.

Zusatz: Sei $R \geqq S$ eine Ringerweiterung. $R / S$ ist dann und nur dann freie $\beta$-Frobenius-Erweiterung, wenn in $R$ Erzeugendensysteme $\left\{r_{i}\right\}$ und $\left\{l_{i}\right\} i=1, \ldots, n$ und $\psi \in \operatorname{Hom}\left({ }_{S} R_{S}, S_{S} S_{\beta S}\right)$ derart existieren, daß

1) $\sum r r_{i} \otimes l_{i}=\sum r_{i} \otimes l_{i} r$ für $\sum r_{i} \otimes l_{i} \in R_{S} \otimes{ }_{\beta S} R$ und alle $r \in R$

2) $\psi\left(l_{i} r_{j}\right)=\delta_{i j}$, wobei $\delta_{i j}$ das Kronecker-Symbol sei.

Beweis: Sei $R / S$ eine $\beta$-Frobenius-Erweiterung. Dann ist

$$
\begin{aligned}
\alpha^{-1}\left(\sum r r_{i} \otimes l_{i}\right)(f) & =\Sigma\left[r^{l} f\right]\left(r_{i}\right) l_{i} \\
& =\alpha^{-1}\left(\sum r_{i} \otimes l_{i}\right)\left(\left[r^{l} f\right]\right) \\
& =\varphi^{-1}\left(\left[r^{l} f\right]\right) \\
& =\varphi^{-1}(f) r \\
& =\alpha^{-1}\left(\sum r_{i} \otimes l_{i}\right)(f) r \\
& =\sum f\left(r_{i}\right) l_{i} r \\
& =\alpha^{-1}\left(\sum r_{i} \otimes l_{i} r\right)(f) .
\end{aligned}
$$

Da das für alle $f \in \operatorname{Hom}\left(R_{S}, S_{\beta S}\right)$ gilt und $\alpha$ ein Isomorphismus ist, ist $\sum r r_{i} \otimes l_{i}$ $=\Sigma r_{i} \otimes l_{i} r$. Weiter ist

$$
\begin{aligned}
r & =\varphi^{-1}\left(\left[r^{l} \psi\right]\right) \\
& =\alpha^{-1}\left(\sum r_{i} \otimes l_{i}\right)\left(\left[r^{l} \psi\right]\right) \\
& =\sum \psi\left(r r_{i}\right) l_{i},
\end{aligned}
$$

also ist $\left\{l_{i}\right\}$ ein Erzeugendensystem von $R$ und $\sum \psi\left(r_{i}\right) l_{i}=1$. Außerdem ist

$$
\begin{aligned}
\beta^{-1} \psi(r) & =\beta^{-1} \psi\left(\sum \psi\left(r_{i}\right) l_{i} r\right) \\
& =\beta^{-1}\left(\sum \psi\left(r_{i}\right) \psi\left(l_{i} r\right)\right) \\
& =\beta^{-1} \psi\left(\sum r_{i} \beta^{-1} \psi\left(l_{i} r\right)\right) \\
& =\beta^{-1} \psi\left(r \sum r_{i} \beta^{-1} \psi\left(l_{i}\right)\right) \\
& =\left[\left(\sum r_{i} \beta^{-1} \psi\left(l_{i}\right)\right)^{r} \beta^{-1} \psi\right](r) .
\end{aligned}
$$

Also ist wegen $\left(\mathrm{ll}^{\prime}\right) \sum r_{i} \dot{\beta}^{-1} \psi\left(l_{i}\right)=1$. Wegen

$$
r=\sum r r_{i} \beta^{-1} \psi\left(l_{i}\right)=\sum r_{i} \beta^{-1} \psi\left(l_{i} r\right)
$$

ist auch $\left\{r_{i}\right\}$ ein Erzeugendensystem. Wegen $\sum \psi\left(l_{i} r_{j}\right) l_{j}=l_{i}$ ist im Falle einer freien Frobenius-Erweiterung $\psi\left(l_{i} r_{j}\right)=\delta_{i j}$. 
Wir beweisen nun die Umkehrung des Satzes. Sei $\Phi(f)=\Sigma f\left(r_{i}\right) l_{i}$ für $f \in \operatorname{Hom}\left(R_{S}, S_{\beta S}\right) . \Phi$ ist ein Homomorphismus. Wegen

$$
\begin{aligned}
\Phi\left(\left[{ }^{l} s r^{l} f\right]\right) & =s \sum f\left(r r_{i}\right) l_{i} \\
& =s \sum f\left(r_{i}\right) l_{i} r \\
& =s \Phi(f) r
\end{aligned}
$$

ist $\Phi$ ein $S$ - $R$-Homomorphismus. Für $r \in R$ ist

$$
\begin{aligned}
\Phi\left(\left[r^{l} \psi\right]\right) & =\sum \psi\left(r r_{i}\right) l_{i} \\
& =\Sigma \psi\left(r_{i}\right) l_{i} r \\
& =r .
\end{aligned}
$$

Für $\Phi(f)=\sum f\left(r_{i}\right) l_{i}$ ist

$$
\begin{aligned}
\beta \psi^{\prime}\left(\sum f\left(r_{i}\right) l_{i} r\right) & =\sum f\left(r_{i}\right) \beta \psi^{\prime}\left(l_{i} r\right) \\
& =\sum f\left(r_{i} \psi^{\prime}\left(l_{i} r\right)\right) \\
& =f\left(r \sum r_{i} \psi^{\prime}\left(l_{i}\right)\right) \\
& =f(r) .
\end{aligned}
$$

Also ist $\Phi$ ein Isomorphismus. Wegen

$$
r=\sum r r_{i} \psi^{\prime}\left(l_{i}\right)=\sum r_{i} \psi^{\prime}\left(l_{i} r\right)=\sum r_{i}\left[l_{i}^{l} \psi^{\prime}\right](r)
$$

schließlich ist $R_{S}$ endlich erzeugt und projektiv, und damit ist $R / S$ eine $\beta$-Frobenius-Erweiterung.

$$
\text { Ist } \begin{aligned}
\left(l_{i} r_{j}\right)=\delta_{i j}, \text { so ist für } 1 & =\sum s_{i} l_{i} \text { mit } s_{i} \in S \\
\sum_{j} \psi\left(r_{j}\right) l_{j} & =\sum_{i, j} \psi\left(s_{i} l_{i} r_{j}\right) l_{j} \\
& =\sum_{i} s_{i} l_{i} \\
& =1 .
\end{aligned}
$$

Weiter ist wegen $\beta^{-1} \psi \in \operatorname{Hom}\left({ }_{S} R_{S}, \beta^{-1} S_{S} S_{S}\right)$ symmetrisch $\sum r_{i} \beta^{-1} \psi\left(l_{i}\right)=1$ erfüllt. Damit ist $R / S \beta$-Frobenius-Erweiterung.

Ist nun $r=\sum s_{i} l_{i}$, so ist $\left[r_{j}^{r} \psi\right](r)=\psi\left(\sum s_{i} l_{i} r_{j}\right)=s_{j}$. Damit ist aber die gewählte Darstellung für $r$ eindeutig, d. h. $R / S$ ist eine freie $\beta$-Frobenius-Erweiterung.

Eine weitere Eigenschaft der Erzeugendensysteme $\left\{r_{i}\right\}$ und $\left\{l_{i}\right\}$ ist

$$
\sum r_{i} \otimes \varrho l_{i}=\sum r_{i} \psi^{*}(\varrho) \otimes l_{i} \text { für alle } \varrho \in Z_{R}(S) \text {. }
$$

Es ist nämlich

woraus (11) folgt.

$$
\begin{aligned}
\alpha^{-1}\left(\sum r_{i} \otimes \varrho l_{i}\right)(f) & =\sum f\left(r_{i}\right) \varrho l_{i} \\
& =\varrho \sum f\left(r_{i}\right) l_{i} \\
& =\varrho \varphi^{-1}(f) \\
& =\varphi^{-1}\left(\left[\psi^{*}(\varrho)^{r} f\right]\right) \\
& =\sum f\left(r_{i} \psi^{*}(\varrho)\right) l_{i} \\
& =\alpha^{-1}\left(\sum r_{i} \psi^{*}(\varrho) \otimes l_{i}\right)(f),
\end{aligned}
$$


Wir führen jetzt die Spur für $\beta$-Frobenius-Erweiterungen ein. Seien die $R$-Rechts-Moduln $A_{R}$ und $B_{R}$ gegeben, sei $R / S$ eine $\beta$-Frobenius-Erweiterung, und sei $f \in \operatorname{Hom}\left(A_{S}, B_{S}\right)$. Es ist bekanntlich $\pi: \operatorname{Hom}\left(R_{S}, B_{\beta S}\right) \cong$ $\cong \operatorname{Hom}\left(R_{S} \otimes_{\beta S} R_{R}, B_{R}\right) \cdot \pi$ ist sogar ein $R$-Rechts-Homomorphismus wegen

$$
\begin{aligned}
\pi\left(\left[r^{l} f\right]\right)\left(r^{\prime} \otimes r^{\prime \prime}\right) & =\left[r^{l} f\right]\left(r^{\prime}\right) r^{\prime \prime} \\
& =f\left(r r^{\prime}\right) r^{\prime \prime} \\
& =\left[r^{2} \pi(f)\right]\left(r^{\prime} \otimes r^{\prime \prime}\right)
\end{aligned}
$$

für beliebige $r, r^{\prime}, r^{\prime \prime} \in R$. Damit ist

$$
\begin{aligned}
\operatorname{Hom}\left(A_{S}, B_{\beta S}\right) & \cong \operatorname{Hom}\left(A_{R}, \operatorname{Hom}\left(R_{S}, B_{\beta S}\right)_{R}\right) \\
& \cong \operatorname{Hom}\left(A_{R}, \operatorname{Hom}\left(R_{S} \otimes_{\beta S} R_{R}, B_{R}\right)_{R}\right) .
\end{aligned}
$$

Der so konstruierte Isomorphismus werde mit $\omega$ bezeichnet. Dann ist

$$
\omega(f)(a)\left(r^{\prime} \otimes r^{\prime \prime}\right)=f\left(a r^{\prime}\right) r^{\prime \prime} .
$$

Setzen wir nun in $R_{S} \otimes{ }_{\beta S} R$ fest $\alpha\left(\varphi^{-1}\right)=\sum r_{i} \otimes l_{i}$ ein, so ist wegen

$$
\begin{aligned}
& \omega(f)(a r)\left(\sum r_{i} \otimes l_{i}\right)=\sum f\left(a r r_{i}\right) l_{i} \\
& =\omega(f)(a)\left(\sum r r_{i} \otimes l_{i}\right) \\
& =\omega(f)(a)\left(\sum r_{i} \otimes l_{i} r\right) \\
& =\sum f\left(a r_{i}\right) l_{i} r \\
& =\omega(f)(a)\left(\sum r_{i} \otimes l_{i}\right) r
\end{aligned}
$$

eine Abbildung $\operatorname{Hom}\left(A_{S}, B_{\beta S}\right) \ni f \rightarrow \operatorname{Spur} f \in \operatorname{Hom}\left(A_{R}, B_{R}\right)$ definiert durch Spur $f=\sum\left[r_{i}^{+} l_{i} f\right]$. Diese Spur-Abbildung ist offenbar im freien Fall identisch mit der dort definierten Spur.

Mit diesen Hilfsmitteln können wir nun die Maschke-Ikeda-Kasch-Charakterisierung von relativ projektiven bzw. relativ injektiven Moduln verallgemeinern.

Satz 11: Sei R/S eine $\beta$-Frobenius-Erweiterung, und sei $A$ ein $R$-RechtsModul. Dann sind die folgenden Eigenschaften äquivalent:

1) $A$ ist $(R, S)$-projektiv.

2) $A$ ist $(R, S)$-injektiv.

3) Es existiert ein $g \in \operatorname{Hom}\left(A_{S}, A_{\beta S}\right)$ mit $\operatorname{Spur} g=\mathrm{id}_{A}$.

Beweis: Die Äquivalenz von 1) und 2) ist in [6] Theorem 20 bewiesen. Wir zeigen, aus 1) folgt 3).

Wir bilden id $\otimes \psi: A_{S} \otimes{ }_{S} R_{S} \rightarrow A_{S} \otimes{ }_{S} R_{\beta S}$. Dann ist

$$
\begin{aligned}
\operatorname{Spur}(\mathrm{id} \otimes \psi)(a \otimes r) & =\omega(\mathrm{id} \otimes \psi)(a \otimes r)\left(\sum r_{i} \otimes l_{i}\right) \\
& =a \otimes \Sigma \psi\left(r r_{i}\right) l_{i} \\
& =a \otimes r .
\end{aligned}
$$

Damit ist $\operatorname{Spur}(\mathrm{id} \otimes \psi)=\mathrm{id} . \mathrm{Da} A(R, S)$-projektiv ist, besitzt $A_{S} \otimes{ }_{S} R$ einen zu $A R$-isomorphen $R$-direkten Summanden $A^{\prime}$. Sei $p$ die Projektion von $A_{S} \otimes{ }_{S} R$ auf $A^{\prime}$, dann ist $p \in \operatorname{Hom}\left(A_{S} \otimes{ }_{S} R_{R}, A_{S} \otimes{ }_{S} R_{R}\right)$. Sei $g^{\prime}$ die Ein- 
schränkung von $p($ id $\otimes \psi)$ auf $A^{\prime}$. Dann ist für $a^{\prime} \in A^{\prime}$

$$
\begin{aligned}
\operatorname{Spur}\left(g^{\prime}\right)\left(a^{\prime}\right) & =\sum g^{\prime}\left(a^{\prime} r_{i}\right) l_{i} \\
& =\sum p(\mathrm{id} \otimes \psi)\left(a^{\prime} r_{i}\right) l_{i} \\
& =p\left(\sum(\mathrm{id} \otimes \psi)\left(a^{\prime} r_{i}\right) l_{i}\right) \\
& =p\left(\operatorname{Spur}(\mathrm{id} \otimes \psi)\left(a^{\prime}\right)\right) \\
& =p\left(a^{\prime}\right) \\
& =a^{\prime}
\end{aligned}
$$

Sei $\mu$ der Isomorphismus von $A$ auf $A^{\prime}$, dann hat $g=\mu^{-1} g^{\prime} \mu$ die gewünschte Eigenschaft, denn es ist für $a \in A$

$$
\begin{aligned}
\operatorname{Spur}(g)(a) & =\mu^{-1}\left(\sum g^{\prime} \mu\left(a r_{i}\right) l_{i}\right) \\
& =\mu^{-1}\left(\sum g^{\prime}\left(\mu(a) r_{i}\right) l_{i}\right) \\
& =\mu^{-1}\left(\operatorname{Spur}\left(g^{\prime}\right) \mu(a)\right) \\
& =a .
\end{aligned}
$$

Da $p$ und $\mu R$-Homomorphismen sind und id $\otimes \psi$ ein $\beta S$-Homomorphismus ist; ist $g \in \operatorname{Hom}\left(A_{S}, A_{\beta S}\right)$.

Um zu zeigen, daß 1) aus 3) folgt, beachten wir, daß $\left\{\sum g\left(a r_{i}\right) \otimes l_{i} \mid a \in A\right\}$ $=A^{\prime}$ ein $R$-Untermodul von $A_{S} \otimes{ }_{S} R$ ist, denn für festes $g$ und $a$ existiert ein Homomorphismus

$$
R_{S} \otimes{ }_{\beta S} R \ni r^{\prime} \otimes r^{\prime \prime} \rightarrow g\left(a r^{\prime}\right) \otimes r^{\prime \prime} \in A_{S} \otimes{ }_{S} R .
$$

Dann ist $\sum g\left(a r_{i}\right) \otimes l_{i} r=\sum g\left(a r r_{i}\right) \otimes l_{i}$. Der $R$-Homomorphismus $\varepsilon$ : $A_{S} \otimes{ }_{S} R \ni \sum a \otimes r \rightarrow \sum a r \in A$ bildet $A^{\prime} R$-isomorph auf $A$ ab. Also ist $A_{S} \otimes{ }_{S} R_{R}=A_{R}^{\prime} \oplus \operatorname{Ker}(\varepsilon)_{R}$, d. h. $A$ ist $(R, S)$-projektiv.

\section{Literatur}

[1] Cartan, H., and S. Emlenberg: Homological Algebra. Princeton Press 1956.

[2] KASCH, F.: Grundlagen einer Theorie der Frobenius-Erweiterungen. Math. Ann. 127, $453-474$ (1954).

[3] - Homologische Algebra, Seminarausarbeitung. Math. Institut der Universität Heidelberg 1959/60.

[4] - Ein Satz über Frobenius-Erweiterungen. Arch. Math. 12, 102-104 (1961).

[5] - Projektive Frobenius-Erweiterungen. Sitzungsber. Heidelberger Akad. 89-109 (1960/61).

[6] - Dualitätseigenschaften von Frobenius-Erweiterungen. Math. Z. 77, 219-227 (1961).

[7] Nakayama, T.: On Frobeniusean Algebras I-III. Ann. Math. 40, 611-633 (1939), Ann. Math. 42, 1-21 (1941), Jap. J. Math. 18, 49-65 (1942).

[8] -, and T. Tsuzorv: A remark on Frobenius-extensions and endomorphism rings. Nagoya Math. J. 15, 9-16 (1959).

[9] - - On Frobenius-extensions I and II. Nagoya Math. J. 17, 89-110 (1960), 19, $127-148$ (1961).

[10] - - Correction to our paper "On Frobenius-extensions II". Nagoya Math. J. 20, 205 (1962).

(Eingegangen am 30. November 1962) 